\title{
Pierre Boulez - Écriture musicale et accident
}

Entretien avec Dominique Jameux

Pierre Boulez et Dominique Jameux

\section{(2) OpenEdition}

Journals

Édition électronique

URL : https://journals.openedition.org/genesis/85

DOI : $10.4000 /$ genesis. 85

ISSN : 2268-1590

Éditeur :

Presses universitaires de Paris Sorbonne (PUPS), Société internationale de génétique artistique littéraire et scientifique (SIGALES)

\section{Édition imprimée}

Date de publication : 20 juin 2010

Pagination : 219-228

ISBN : 978-2-84050-697-3

ISSN : 1167-5101

\section{Référence électronique}

Pierre Boulez et Dominique Jameux, «Pierre Boulez - Écriture musicale et accident », Genesis [En ligne], 30 | 2010, mis en ligne le 17 mai 2013, consulté le 30 mars 2023. URL : http:// journals.openedition.org/genesis/85; DOI : https://doi.org/10.4000/genesis.85 


\title{
Écriture musicale et accident Entretien avec Dominique Jameux
}

\author{
Pierre Boulez
}

La figure de Pierre Boulez, compositeur majeur de l'après-guerre, puis chef d'orchestre de dimension internationale depuis les années soixante-dix, est celle d'un créateur qui s'est toujours penché sur sa création, et la création musicale en général. L'image sommaire qu'on a longtemps présentée de lui (un musicien-mathématicien, rationaliste, théoricien du "degré zéro de l'écriture musicale ", planificateur dodécaphoniste de ses æuvres) semblait ne pas laisser place à celle d' un compositeur naturellement en proie au doute, aux résistances du matériau, à l'impasse créatrice à surmonter, aux survenues de l'"accidentel » dans le processus de création. Il n'en est évidemment rien - une fois rappelé (ce qui vient en l'occurrence en fin d'entretien) que la musique, plus que tout autre art, est étroitement dépendante de ses conditions de réalisation : niveau des interprètes, temps de répétition, etc. La notion d" "accident " y est donc pertinente tant pour l'amont de l' œuvre (sa genèse, qui reste souvent opaque) que pour son aval (son inscription dans le monde du concert). La reprise, les différentes versions des æuvres, l' inachèvement même, constituent par ailleurs pour partie le catalogue de l'œuvre de Pierre Boulez en matériau abouti d'esquisses. L'entretien qui suit ne constitue que l'embryon d'une réflexion sur ce sujet. On trouvera à la suite de l'entretien les références sommaires des œuvres évoquées durant celui-ci.

Dominique JAmeux - Nous parlons de la notion d" " accident ». Si vous voulez bien, j'aimerais repartir de vos expressions " par volonté et par hasard», et « le hasard devenu nécessité », comme définissant la création.

PIERRE BOULEZ - « Par volonté et par hasard », certainement, cela a trait surtout à l'éducation et aux décisions que l'on prend en fonction de l'éducation que l'on reçoit, parce qu'on a rencontré certaines personnes, à un certain moment de son existence, et pas à un autre moment. Ainsi, même dans la volonté, il y a déjà beaucoup de hasard, à vrai dire. Mais l'important est ce qu'on en fait : j'ai dit surtout, par cette expression, qu'il faut être autodidacte par volonté et non par hasard. C'était très précis. Être autodidacte, parce que je pense qu'il n'y a pas d'éducation véritablement transmissible - en ce qui concerne en tout cas la composition. C'est un héritage que chacun absorbe en particulier, choisit seul, et tire des leçons vraiment personnelles et non pas des leçons générales. Dans la musique classique, par exemple, ce n'est pas le schéma tonal qui va vous rendre justice, qui va rendre justice du moins aux ouvres qu'on analyse. Il est, en particulier dans les fugues de Bach, très déterminé, prédéterminé même. Il peut y avoir des exceptions, les fugues ne sont pas toutes exactement sur le même modèle, mais enfin le modèle général est là. En particulier dans les fugues : le sujet, la réponse, le contre-sujet, différents contre-sujets, les combinaisons contrapuntiques, tout cela est naturellement maîtrisé par des lois, et en fonction de certaines lois. Maintenant, ce qu'on en fait, c'est autre chose !

\section{Lois et accident}

\section{J. - Des lois ou des habitudes?}

$P$. B. - Non, ce sont des lois. Ce que j'appelais des « lois de responsabilité ». Quand vous écrivez un sujet, puis un contre-sujet, le contre-sujet a une sorte de responsabilité harmonique ou contrapuntique vis-à-vis du sujet. De toute façon, vous apprenez ces lois, celles de l'harmonie, du contrepoint... Bien sûr, vous pouvez élargir ces lois, vous pouvez les rendre caduques ou, en tout cas, les rendre plus 
efficaces dans un certain ordre d'idées, par l'emploi d'intervalles déterminés. On peut dire que la façon dont Wagner utilise l'harmonie tonale est vraiment beaucoup plus riche ou beaucoup plus étendue que celle de Mozart : ce qui n'empêche pas celle de Mozart d'être très efficace et de convenir parfaitement à son vocabulaire. Donc, il y a des lois plus ou moins larges, plus ou moins efficaces, plus ou moins libres aussi, mais ce sont des lois. Et ce que l'on peut appeler l'accident, par rapport à cela, c'est simplement si l'on détourne la loi de sa fonction ou si on la refuse. Le vocabulaire harmonique académique, par exemple, a pendant très longtemps refusé les « intervalles parallèles ». Bon, très bien : il a suffi que Debussy emploie des accords parallèles pour que ces lois soient détournées de leur emploi, et que l'harmonie ne soit pas uniquement fonctionnelle mais produise simplement un objet sonore identifiable. D'une loi, on est passé à une autre loi, parce qu'on a utilisé le mot «accord » dans un autre sens. Hasard, nécessité, volonté : il y a beaucoup d'espace autour de ces notions.

\section{«Invente la règle, puis suis-la ! »}

D. J. - J'ai l' impression que dans votre vision de l'éducation et de l'apprentissage, vous êtes très "maître chanteur », de Wagner, du moins très "Hans Sachs ». Pour être efficace, pour être transmis, le langage doit passer par un certain nombre de lois, par exemple celles du «Bar»-deux énoncés identiques, un troisième différent, les deux énoncés identiques étant plus ou moins les parents de l'énoncé différent, dans une sorte de fantaisie familiaresque! - et l' on peut enseigner ces lois : Sachs donne une leçon de musique à Walther dans cette perspective, et Walther apprend ces lois, les accepte, les intègre... Mais dans son chant de concours, s'il commence en les suivant, à un moment, il dérape, il y a un "accident», il ne suit plus les règles académiques $d u$ «Bar». Son Chant de maîtrise est en fait un mauvais Bar. C' est l'accident qui a en quelque sorte tordu la loi.

P. B. - Oui, c'est un affranchi ! Je ne me rappelle pas le texte des Maîtres chanteurs littéralement, mais je pense que la première rencontre de Walther avec Sachs est vraiment la glorification de l'anarchie, dans un certain sens : l'im- provisation rachète tout, l'inspiration rachète tout. Alors, face aux Maîtres chanteurs qui ne font confiance, au contraire, qu'à la loi et à l'organisation, Walther tient compte de l'organisation, mais s'en affranchit et la dépasse. Il ne dérape pas, il la dépasse. Et on le reconnaît alors comme vainqueur précisément parce qu'il a su inventer un vocabulaire à partir des anciennes lois mais en leur donnant un horizon différent.

D. J. - Reprendriez-vous à votre compte la consigne de Sachs à Walther : "Invente la règle, puis suis-la. »?

P. B. - Un peu, oui. C'est-à-dire on la suit, mais d'une façon personnelle. Quelquefois, certains compositeurs établissent un parcours a priori, quasiment avant les idées... Ils partent d'une idée, puis remplissent le parcours, comme des boîtes : tel développement, puis tel développement... Je n'arrive pas à faire ainsi, parce que les idées constituent un organisme vivant, qu'on ne peut placer n'importe comment, n'importe où, et surtout dans du préfabriqué ! L'essentiel, pour moi, est de regarder comment ces organismes vivants se développent, et s'ils se développent quelquefois, non pas monstrueusement - je n'irai pas jusque-là - mais d'une façon imprévue, en tout cas de vous, tout en étant dans leur propre logique. Vous créez des objets qui finalement vous imposent leur propre logique au lieu que vous leur imposiez celle que vous aviez prévue. C'est ce que j'appellerai « l'accident ».

\section{Le texte}

D. J. - Est-ce que la présence d' un texte modifie la situation au départ?

P. B. - Le texte sert de support au départ. On peut noter quelques idées... Mais cela ne m'est pas arrivé très souvent. J'ai fait seulement trois œuvres... quatre œuvres, avec texte. Dont Char, trois fois : Soleil des eaux, Visage nuptial et Marteau sans maître.

D. J. - Mallarmé...

P. B. - Mallarmé aussi, une fois. 
D. J. - Une fois de façon directe, dans Pli selon pli, et une fois indirecte dans La Troisième Sonate.

P. B. - Oui, c'est vrai, La Troisième Sonate est inventée à partir de Mallarmé, mais j'ai découvert le Livre après l'avoir écrite.

\section{J. - Mais vous aviez lu Igitur avant.}

P. B. - Oui. Et après il y a eu simplement un tout petit épisode avec Cummings. Point final. Donc je suis resté quelquefois des années sans écrire avec texte... Ce n'est pas un problème qui $\mathrm{m}$ 'a préoccupé constamment, comme certains. Par exemple Berio revient très souvent à un texte littéraire. Moi pas, probablement parce que je trouve un texte très contraignant. Il vous impose une sorte d'architecture. Ce qui est intéressant d'ailleurs, c'est d'essayer d'avoir une architecture, soit vraiment parallèle au texte littéraire, soit qui au contraire le contredise, ou en tout cas contrepointe. Cela m'intéresse aussi, moins cependant que l'architecture pure de la musique, si je puis dire.

D. J. - Mais la rencontre avec un texte, qui donc en ce qui vous concerne est intervenue une petite demidouzaine de fois, est-elle en elle-même un "accident», quelque chose d' un peu imprévisible : vous lisez un texte tout d'un coup, il vous émeut, vous touche, vous intéresse, vous passionne - est-ce un accident? Et une fois que vous avez entrepris une ouvre à partir de ce texte, ce texte au contraire n'empêche-t-il pas un peu l'accidentel de se produire?

P. B. - Non, il le provoque. Il peut le provoquer, en tout cas. Quoique pour Mallarmé, par exemple, il est très difficile de ne pas regarder la structure du texte, parce que c'est vraiment le point de la poésie - française en tout cas - où le formalisme a été le plus développé, le plus contraignant. Que fallait-il alors faire ? Essayer - à cette époque-là, il y a longtemps déjà ! - de trouver une structure qui définisse par des critères musicaux ce qu'est formellement le sonnet : des rimes - rimes féminines, rimes masculines, qui alternent ou bien qui sont groupées, et les deux quatrains, les deux tercets, etc. Il y a de nombreuses règles formelles qui sont extrêmement contraignantes. À partir de là, est-ce que mon architecture va se baser sur les quatrains ou sur les tercets ou bien sur les rimes ? Va-t-elle tenir compte du nombre de syllabes ? Etc., etc.

D. J. - Je note que dans ces différentes hypothèses, il y en a une qui n'apparaît pas, c'est le sens du texte.

P. B. - Si. Via la symbolique des instruments, qui est choisie par rapport au texte. En particulier pour la Deuxième Improvisation, « une dentelle s'abolit ». Le mot « dentelle » est transmis d'abord par des arabesques très décoratives. Le mot suscite la couleur. Il s'agit d'un reflet dans un miroir. Le miroir est transcrit par des surfaces lisses qui sont les instruments à résonance métallique. Donc il y a une symbolique, non seulement par rapport au sens, mais une symbolique directe du choix des instruments par rapport au sens ou du choix de l'écriture musicale par rapport au sens.

D. J. - Mais si je pense à la pièce terminale, «Tombeau», qui parle du jugement de la postérité, du «peu profond ruisseau " qu'il faut traverser pour aller de l'autre côté...

P. B. - Il s'est agi d'une circonstance particulière, la mort d'un mécène du festival de Donaueschingen. On m'avait demandé de lui faire un hommage, puisque le Domaine musical était invité, juste cette année-là. Je l'ai fait, mais j'ai vu ensuite que ces idées d'hommage funèbre pouvaient s'agrandir, et à ce moment-là, comme je travaillais dans Mallarmé, j'ai trouvé ce « peu profond ruisseau calomnié » et là, il est évident que j'ai élargi complètement l'objet qui a été à la naissance de ce Tombeau. Vous savez, les idées naissent comme cela, peut-être quelquefois même d'une façon très simpliste. Je voulais vraiment élargir le Tombeau et citer le vers de Mallarmé - non pas comme j'avais fait dans les Improvisations, utiliser les poèmes en entier, mais utiliser seulement $u n$ vers. Puis il y a eu la dédicace, la première pièce : là au contraire, je voulais que la citation fût tout à fait au début et que l'on sache que la pièce se place sous l'égide de Mallarmé, et qu'elle préside à son développement. 


\section{L'ange en filigrane}

D. J. - Mais que devient l' accident, dans ce contexte? Parce que vous semblez ici presque définir une sorte de grande trajectoire de l'ouvre, établir de grands plans de l'auvre...

P. B. - Oui, mais qui s'est réalisé au fur et à mesure et en arrière. Il y avait d'abord les trois Improvisations (plutôt deux, puis une troisième). Ensuite il y a eu Tombeau qui est venu fortuitement, comme un objet supplémentaire. Et pour faire l'équivalent de Tombeau, j'ai écrit Don. L'architecture apparaît très organisée, mais elle est tout de même accidentelle, parce qu'elle n'était pas préméditée. Quelquefois j'ai des idées très précises sur ce que je veux faire, quelquefois j'avance... pas dans le noir bien sûr, mais quand même dans l'ombre et dans le doute et je ne trouve pas... Je ne suis pas du reste à la recherche de «la Vérité », je pense qu'il n'y a pas de vérité. Je suis à la recherche d'une sorte d'architecture de la pensée, de la sensibilité et de la narration. Et cela se découvre au fur et à mesure que l'œuvre avance. Par exemple dans Dérive I, il n'y avait pas de doute que cette œuvre était finie comme elle est, et je ne songe pas du tout à la retoucher, même vingt ans après. Mais Dérive II m'a pris très longtemps : je voyais que cette œuvre avait besoin d'être développée, je ne savais pas comment. Alors, on laisse sur le côté pour le moment et puis il y a d'autres idées qui viennent, d'autres œuvres même, qu'on a composées entre-temps. Tout s'additionne au fur et à mesure et l'architecture définitive se présente comme une espèce de zigzag. Il y a un texte très peu connu de Henry Miller, qui s'appelle « Je porte un ange en filigrane ». Il dit : je veux dessiner un ange et je finis par dessiner un cheval. Je trouve que c'est assez symbolique - pour moi en tout cas - de ma démarche.

D. J. - Pour terminer sur Pli selon pli, j' ai l'impression qu'il y a là de l'accidentel - notamment la genèse de l' 'uvre, cette marche a retro de l'œuvre... - lequel résulte aussi des commandes, des événements, la mort de X... et que cet accidentel produit du non accidentel.

P. B. - Oui, absolument. Parce que quand on organise à ce moment-là, on le fait en fonction de ce qu'on rencontre, puis on réfléchit, et on se dit : « Comment vais-je l'intégrer? » Et c'est évident que rien n'est disposé comme cela simplement parce qu'on est levé à six heures du matin ou parce qu'on est de mauvaise humeur ou de bonne humeur ! Il y a une organisation profonde qui reste en vous, même inconsciemment, même si vous n'y songez pas constamment. Ce travail se fait dans l'inconscient. De cela, je suis persuadé. Vous trouvez quelquefois des solutions auxquelles vous n'avez pas pensé, vous ne les cherchez pas, mais vous les trouvez.

\section{Genèse et accident}

D. J. - Prenons l' idée de la genèse d' une æuvre. Vous l'entreprenez, j' imagine, à partir d' une incitation qui reste plus ou moins mystérieuse. Il y a un point de départ, une étincelle, il y a quelque chose qui vous sollicite dans une direction, et à ce moment-là vous n'avez pas de plan préconçu sur l'œuvre...

P. B. - Non, ça dépend. Je ne tiens pas forcément à me rappeler la genèse d'une œuvre. Mais si je pense à celle de Répons, je vois cette œuvre que j'avais faite pour Donaueschingen en 1968, sur un poème de Michaux, en 1958 je veux dire... Poésie pour pouvoir. C'était un premier essai de réalisation avec la technologie, mise en relief avec trois orchestres différents, ou trois groupes différents exactement, mais seulement avec deux chefs, dont un groupe était flottant, etc. Bon, cela n'avait pas été réussi, d'abord parce que la technologie n'était pas assez avancée à l'époque, deuxièmement parce que $\mathrm{j}$ 'avais fait cette partition trop vite, très pressé par le temps. Ce n'était donc pas satisfaisant, mais j'avais gardé cette idée en tête : utiliser l'espace et la technologie. Comme j'avais l'Ensemble intercontemporain à ma disposition, je me suis dit que j'allais tenter Répons avec un ensemble plus petit que l'orchestre symphonique, avec lequel les résultats peuvent être plus convaincants plus rapidement. Et au lieu d'avoir des groupes d'orchestre qui étaient divisés, j'ai pris des solistes - il est beaucoup plus facile de travailler avec des solistes, d'utiliser la technologie avec eux qu'avec des grands groupes. Donc j'ai profité des leçons de l'échec. Mais entre Poésie pour pouvoir et Répons, il y a eu cette intention, qui a mis de 1958 à 1981 pour se réaliser ! Et encore, la version finale date de 1984. 
Ainsi, on voit quelquefois que cela chemine très lentement. Au contraire, la première version d'Éclat (pas Éclats multiples, la première version d'Éclat), c'était tout de suite un groupe d'instruments, d'instruments résonants, depuis la mandoline - l'instrument qui résonne une demiseconde - jusqu'au piano dans le grave qui peut résonner jusqu'à quinze secondes à peu près. Et à ce moment-là je me suis dit - parce que le problème du temps en musique est vraiment quelque chose qui me préoccupe beaucoup, c'est un paramètre essentiel - qu'avec ces instruments, je n'avais pas besoin de mesure du temps a priori. La mesure du temps, ce sera leur capacité de résonance. Cela, c'est un premier point : quand on laisse les instruments résonner, on peut les analyser au fur et à mesure et donc on ne dépend pas d'un mètre, ou d'une métrique, on peut être tout à fait libre. Et le second point en découle : si je ne laisse pas ces instruments résonner, si je fais des combinaisons différentes, mais très sèches, je ne peux absolument pas les analyser. J'entends un combiné de timbres, mais je suis incapable de l'analyser. Je peux donc aller de l'inanalysable au totalement analysable. Et j'ai commencé à penser là-dessus. D'autres choses se sont greffées, cela ne suffisait naturellement pas pour faire une œuvre. J'ai commencé à ce moment-là à faire interférer aussi la dimension libre et la dimension obligée, c'est-àdire une succession d'événements qui se placent toujours dans le même ordre et une succession d'événements qui peuvent au contraire se situer dans n'importe quel ordre, suivant que l'on donne telle directive ou telle autre. Donc vous voyez que cela s'est accumulé.

Mais pour Explosante fixe, je me souviens en particulier qu'il y avait d'abord six instruments, qui étaient complètement indépendants, que la technologie était complètement impuissante à organiser. C'était beaucoup trop compliqué, et à ce moment-là j'ai changé mon fusil d'épaule. J'ai pris un instrument soliste et pour les autres, je les ai soumis à une loi ordinaire, mais il n'y avait que l'instrument soliste qui transgressait par la technologie son domaine propre. Ainsi vous voyez, quelquefois, quand vous rencontrez des difficultés, vous êtes obligé de trouver des solutions différentes, et c'est comme cela que vous avancez... Mais quelquefois la solution vient dans un éclair, si je puis dire : je me souviens que pour Constellation miroir, j'ai pensé tout de suite à la structure, comment cela pourrait être. Pour ma Troisième Sonate elle-même - bien qu'elle ne soit en principe pas finie, mais elle a été finie : parce que les pièces étaient trop courtes, je veux les allonger - j'ai conçu les cinq mouvements vraiment immédiatement, avec chaque fois une sorte de technique, un peu suivant le projet de Joyce : que chaque mouvement ait sa propre technique, sa propre technique d'utilisation de développement formel, etc. C'est venu d'un seul coup, mais c'était une des rares fois où j'ai eu un projet qui s'est imposé comme cela si rapidement.

D. J. - On voit bien que l'accident peut débloquer une situation, peut orienter différemment un processus, peut donner, esquisser une idée à laquelle on n'avait pas pensé avant... Tout ça c'est ce que vous appelez des accidents heureux. Mais dans ces cas-là, est-ce qu'il existe des accidents malheureux?

P. B. - Non, je ne pense pas. Pas avec moi en tout cas. Si je considère 1'accident, cela me force à réfléchir. C'est pour cela que je le suscite presque... Je l'ai dit dans un article : j'aime beaucoup les recherches purement techniques, parce qu'elles sont dégagées de la mémoire. Par exemple j'ai fait - avant Dérive II - des essais sur les périodicités, parce que j'avais vu, dans Ligeti en particulier, à partir de son Trio et des œuvres plus récentes, qu'il utilisait d'une façon encore assez visible les différents types de périodicité : périodicité de cinq superposée à une périodicité de quatre ; les modulations de tempo de Carter par exemple, qui m'ont également intéressé. Cela m'a donné l'idée de penser aux différentes possibilités, justement, de superposer différentes périodicités. Après ça, j’ai fait des « études »- que j’ai conservées comme études, vraiment, même pas des esquisses : des études de faisabilité, si je puis dire. Des études d'ingénierie, si on veut. Et à partir de là, vous trouvez des solutions auxquelles vous n'avez pas pensé, vous n'y auriez pas pensé autrement. Je me méfie, même si je l'accepte aussi, parce que je ne peux pas non plus seulement m'en méfier, je me méfie de la mémoire. Parce que vous pensez trouver quelque chose, qui est inédit, qui est bien, qui va servir... en fait vous re-servez quelque chose que vous avez déjà utilisé et que votre mémoire vous fournit, parce que c'est très commode, c'est facile et vous vous dites, « je le place dans un contexte 
différent, donc ce sera très bien ». En fait, la mémoire vous fournit des bribes, quelquefois du reste un peu distordues, mais la mémoire agit - heureusement du reste que vous en avez une, parce que vous savez ce que vous avez fait, vous savez d'où vous venez tout simplement - mais quelquefois j'aime les recherches purement techniques de cet ordre-là, même sur les intervalles, sur les accords, pour voir comment on peut catégoriser les accords, pour voir si on reconnaît certaines échelles d'intervalles, etc. Et à partir de là, oui, je vois ce que cela peut produire, souvent des résultats auxquels je n'aurais pas pensé autrement. Donc, je suscite vraiment la résistance de ce matériau à ma mémoire.

D. J. - Le système sériel au départ, c'est-à-dire dans la pensée de Schönberg, n'était-il pas en quelque sorte fabriqué pour éviter l'accident?

P. B. - À mon avis, le système de Schönberg avait quelque chose de très incomplet. Il y avait toujours une forme obligée, et aucune possibilité d'échapper à une succession donnée. Or, ce qui est intéressant, c'est d'être libre de la succession, même dans une hiérarchie qui s'organise strictement. C'est l'opposition entre écriture libre et écriture obligée : Bach a écrit des canons extrêmement complexes - dans L'Art de la fugue, en particulier - mais il a écrit aussi des préludes qui sont d'une grande liberté, du point de vue harmonique comme du point de vue mélodique. Et plus Schönberg a progressé, plus il s'est contraint à utiliser d'abord ce chiffre douze, qui est tout de même obsessif, et qui l'oblige toujours à un certain nombre de combinaisons.

D. J. - Mais qui vient du système tempéré, tout simplement.

P. B. - Oui, d'accord, mais moi aussi j'utilise le système tempéré : mais avec quelquefois quatorze notes, sept, treize ou Dieu sait combien? Pourquoi vouloir toujours utiliser les douze sons? Parce que, surtout, quand on voit la façon dont il les a utilisés, c'est pratiquement toujours deux fois six : cela le contraint à des gestes où sa liberté est vraiment très menacée !

\section{J. - Webern aussi : six plus six.}

P. B. - Webern, c'est plutôt quatre fois trois, ou trois fois quatre, et il l'utilise très sciemment avec des formes, comme des formes géométriques : je pense à certains tableaux de Kandinsky ou de Klee. Vous avez par exemple, comme objets de variation, des triangles, des cercles, des carrés, ou bien des ovales, peu importe, et il utilise ces formes géométriques très précisément. Webern, par exemple, dans le Concerto Opus 24, utilise un objet de trois sons qui revient quatre fois, etc., avec les variations rythmiques. Et à un moment, il le déplace : dans le développement du premier mouvement, après justement cette espèce d'extrême rigueur de quatre fois trois, à ce moment-là il déplace et utilise cinq sons contre trois, etc. Mais si je comprends pourquoi ils ont dû en passer par là, on n'était pas obligé d'y rester. C'est surtout cela que je veux dire.

D. J. - Est-ce que vous dites cela en pensant à votre propre expérience dans les années 1953-1954, où non content d'organiser les hauteurs selon la grammaire dodécaphonique, vous avez organisé l'ensemble des paramètres de cette façon-là ? C'est une phase qui n' a pas duré très longtemps, mais que vous avez quand même traversée.

P. B. - Oui, j'ai été intéressé, parce que je me disais... si on organise une dimension du son de cette façon-là, il n'y a aucune raison pour que les autres dimensions du son ne soient pas organisées pareillement. Voyons ce que cela va faire. Ma Structure I, surtout la Première pièce 1a (elle dure trois minutes), c'était justement pour voir jusqu'à quel point l'automatisme peut aller sans que le compositeur intervienne. Je n'y ai pas attaché plus de prix qu'à une expérience... C'est un peu le carré blanc de Malevitch, si vous voulez.

D. J. - «Sérialisme généralisé », disait-on à l' époque. Donc avec une sorte d'anéantissement de l'idée même d'accident?

P. B. - Absolument. Ou alors tous les accidents sont permis. Si j'avais eu par exemple un ordinateur à ma disposition en 1951, j'aurais volontiers essayé : je fournis ces données-là à la machine, qu'est-ce qu'elle en fait ? 
C'est-à-dire que l'auteur n'existe plus. J'avais dit du reste que ce qui m'intéressait, c'est d'arriver à l'anonymat de l'auteur. Et vous savez le titre que je voulais donner à cette structure ? À la limite du pays fertile, et je savais très bien de quel côté était la limite, par-dessus le marché !

D. J. - Au demeurant, il y a des différences de densité, dans cette Structure 1a, qui font qu'il y a tout de même une sorte de parcours, peut-être involontaire...

P. B. - Non, non, il y a un parcours déterminé, ce que j'appelle l'enveloppe, parce que j'ai toujours considéré que la forme doit être dans une sorte d'enveloppe, qui est saisissable. Alors, il y a en effet - mais c'est extrêmement rigoriste - une densité qui va de un à six, tout simplement. Donc en effet par moments un élargissement, puis un rétrécissement. On entend plus facilement, ou moins facilement : malgré tout je me basais encore sur la perception - et je me base toujours sur la perception.

\section{Mémoire et réminiscence}

D. J. - Vous parliez tout à l' heure de la mémoire, dont vous vous méfiez en partie mais dont vous reconnaissez qu' elle existe. Vous êtes chef d'orchestre, et avez dirigé à peu près toute la musique $d u X^{e}$ siècle disponible. N'y a-t-il pas, pour un créateur chef d'orchestre, le danger de la réminiscence - problème qu'a bien connu Mahler, par exemple. Avez-vous rencontré ce problème-là, y a-t-il occasionnellement des bouffées d' une musique qui n'est pas de vous, et qui fait irruption sur votre papier à musique?

$P . B$. Non, parce que c'est déjà trop distant. Il n'y a rien à faire. Ce qui m'intéresse quand j'entends une musique - j'ai toujours été comme cela, de toute façon, chef d'orchestre ou pas - c'est d'essayer de voir le noyau de cette musique. Par exemple, lorsque j'ai entendu, je m'en souviens, la musique du gagaku, la musique de cour japonaise, qu'est-ce qui m'a intéressé ? Pas d'en reprendre les instruments, ou la sonorité ; mais la dimension du temps. C'est quelque chose de très abstrait, mais c'est cela qui m'a intéressé en musique. Un temps extrêmement distendu, où il n'y a pas moyen de se repérer dans une métrique quelconque : il n'y a pratiquement ni début ni fin - en tout cas pour un étranger comme moi, qui ne connaît pas les lois du gagaku. Je ne les ai pas étudiées, mais je me basais sur mes impressions et sur la perception que j'en avais. Oui, cela, ça m’intéresse.

S'agissant de Mahler, que j'ai beaucoup dirigé (spécialement ces dix dernières années), qu'est-ce qui m'intéresse ? Ce n'est pas le style de Mahler - non pas que cela m'irrite, mais c'est simplement loin de moi, en tant que sensibilité - c'est la façon dont il utilise le pouvoir narratif de la musique et qu'il utilise une structure qui est très ferme, très solide et très organisée, mais qui possède un pouvoir narratif très libre, en particulier dans la grande durée. Et je dois dire, Dérive II ne serait pas de cette dimension, pas plus que Sur incises ne serait né, si je n'avais eu en face de moi ce geste narratif qui est vraiment très important. Donc vous voyez que cela n'a rien à voir avec l'imitation ou le style, mais avec quelque chose de beaucoup plus profond.

D. J. - Diriez-vous qu'il y a des compositeurs qui sont plus... comment dire? non pas accidentels, mais qui font plus que d'autres appel à la notion d'accident dans leur rhétorique? Beethoven, par exemple.

P. B. - Oui, c'est ce que j'allais vous dire immédiatement : si vous comparez Chopin à Beethoven, dans Chopin, il n'y a pas beaucoup d'accidentel. Dans beaucoup de productions : les valses, les mazurkas - même si les mazurkas ont par moments un grand intérêt - du point de vue formel, c'est très facile de s'y repérer et dans toute sa vie (qui n'a pas été très longue, je l'avoue) il n'a pas beaucoup changé de cadre d'action de ce point de vue-là. Bon, si l'on va par exemple dans les Ballades, elles sont beaucoup plus intéressantes.

D. J. - Ou La Barcarolle, par exemple, dont la forme n'est pas si évidente que cela...

P. B. - Oui, mais ce n'est tout de même pas son point le plus fort. C'est une musique que j'aime énormément, mais pas pour son aspect formel. Pour le sens de l'arabesque, de la décoration, pour sa sensibilité harmonique, qui est extrê- 
mement aiguë, pointue et intéressante - spécialement dans les mazurkas, d'ailleurs, influencées par la musique populaire ; il y a des glissements harmoniques qui sont quasi préwagnériens ! Un de ses grands chefs-d'œuvre, ce sont les Préludes, mais pas davantage pour la forme, pour beaucoup d'autres raisons. Par contre, dans Beethoven, si vous voyez l'Opus 106 ou l'Opus 111, ou les derniers quatuors, il y a à la fois une évidence de la forme et en même temps une complète imprévisibilité. Si vous voyez la Grande fugue, on se dit : comment a-t-il pu concevoir ça? L'utilisation des motifs, l'utilisation du tempo, l'utilisation de la densité, etc. Parce qu'il y a des moments où vous êtes encore surpris par la façon dont les développements s'enchaînent ou ne s'enchaînent pas, et s'opposent assez brutalement.

D. J. - Beethoven, comme chacun sait, était un compositeur à esquisses et à ratures. Est-ce que la rature est une fenêtre d'accès pour comprendre l'accident heureux?

P. B. - Je pense que oui : si l'on étudie les manuscrits, certainement on voit comment les choses s'organisent, comment une idée se transforme. Mais même dans cette phase-là, chez quelqu'un non pas de primesautier mais d'instinctif, on voit comment par exemple Stravinsky - je parle bien d'une période déterminée, celle du Sacre par exemple - a transformé des mélodies populaires qui n'avaient pas grand intérêt en soi, et comment, à partir des mêmes intervalles, il a commencé à faire des choses extraordinairement frappantes et qui rejettent le matériau originel. Il suscite l'accident pour transformer quelque chose qui n'est pas banal, mais qui certainement lui parlait et qu'il a transformé en son propre objet.

\section{Projet, imprévu, réalisation}

D. J. - Je voudrais revenir à votre ouvre, par exemple à une auvre comme Sur incises. D'abord je crois que Sur incises s'est développé dans une direction qui était totalement imprévisible au départ...

P. B. - Oui, j'ai commencé par la fin et réalisé le début après coup. Grosso modo, il y a deux parties dans la pièce
Incises elle-même : une partie introductive, qui est très courte, suivie d'une toccata, si l'on peut dire, parce que c'était au départ une pièce de virtuosité, qui est aujourd'hui beaucoup plus longue. Que s'est-il passé ? Je me suis dit que la partie toccata, je m'en occuperai après, simplement pour la développer, pour voir comment elle se développe. J'ai commencé par la partie qui n'était pas développée ; j'avais l'intention d'abord de faire un concerto de piano, parce que Pollini et Berio avaient commandé cette œuvre pour un concours de piano : je me suis dit que ce serait intéressant, non pas de faire un concerto dans le sens classique, mais de faire une œuvre vraiment pour piano principal et ensemble ou orchestre, je ne savais pas. Et finalement, quand j'ai commencé à travailler sur l'introduction, j'ai vu que cela me fournissait énormément d'idées, de sonorités, etc., et que ce serait plus intéressant d'avoir des perspectives entre plusieurs pianos, d'avoir probablement un piano principal et deux pianos ombres, si je puis dire, deux pianos échos. Finalement, au fur et à mesure que j'ai réfléchi et avancé dans l'écriture, j'ai vu que ces trois pianos étaient absolument de même niveau, que l'un avait peut-être plus tendance à être dans le grave, l'autre à être dans l'aigu, mais que les trois étaient d'égale force. Je me suis alors dit que c'était impossible de faire un concerto comme ça, cela n'avait aucun intérêt, il fallait donc que je trouve un ensemble qui se base sur des sonorités résonnantes, et quelle est la meilleure combinaison? Une combinaison harpe, piano et percussions-clavier (clavier résonnant). Donc, j'ai travaillé sur cette introduction, qui est devenue la grande partie de la pièce ; et après, la partie toccata, je l'ai mise en exergue, si je puis dire, en la multipliant naturellement, en me servant de la multiplication que me fournissaient les trois trios présents, qui pouvaient aussi bien être trio de trois harpes, trio de trois pianos, trio de percussions ou bien trois trios de harpespianos-percussions.

D. J. - Justement, parlons un peu de cette toccata : elle occupe maintenant une place importante, en durée, dans l' euvre, on pourrait imaginer que c'est, soit en termes musicaux traditionnels une sorte de mouvement perpétuel, soit, en termes visuels, comme une frise sonore, de ruban sonore assez opaque, assez...j allais dire presque uniforme, structurellement très stable au point de vue sonore, qui se 
déroule à grande vitesse et qui de temps en temps est interrompue par des petites enclaves... Est-ce que ces enclaves peuvent donner l'impression que ce sont des « accidents» dans le ruban?

P. B. - Ce sont des accidents prévus, en tout cas. J'avais déjà une continuité au départ. Au bout d'un certain temps, quand on réfléchit, on se dit : il faut que je mêle les deux gestes : geste continu, geste discontinu ; geste très métrique (dépendant de la métrique) et geste indépendant de la métrique et faisant davantage appel aux qualités proprement sonores. C'est comme ça que cette interaction est intervenue entre deux façons de mettre la musique « en gestes », si je puis dire.

D. J. - Vous parliez il y a un instant de Poésie pour pouvoir, où la technologie électro-acoustique n'était pas développée au point de vous permettre d'avancer. Est-ce que mutatis mutandis, vous diriez que c'est à peu près le même type d'entraves que vous avez rencontrées avec Visage nuptial, lorsque vous vous êtes rendu compte à un moment que l'orchestration première de la pièce, par rapport à ce que vous connaissiez alors de l'orchestration (notamment des grandes partitions que vous aviez dirigées entre-temps), vous rendait insupportable la version première?

P. B. - Oui, absolument. Une fois, je suis arrivé, j'ai commencé une répétition et au bout d'une demi-heure, trois quarts d'heure de répétition, j'ai dit non, arrêtez, ce n'est pas possible ! Et c'est à ce moment-là que j'ai pris la décision de refaire l'orchestration complètement; d'abord pour des raisons pratiques, parce que la rythmique était notée d'une façon extrêmement difficile à lire, deuxièmement parce qu'il y avait des choses qui pouvaient arriver seulement après trois heures de répétition et qui risquaient de ne pas arriver non plus, même alors ! Troisièmement : certaines choses que j'avais cru possibles étaient tout à fait aléatoires ; j'ai remplacé ces choses très aléatoires par des choses vraiment possibles, ce qui impliquait de transformer complètement l'écriture. Par exemple, tout ce qui était écrit en quart de tons aux cordes, je l'ai remplacé par des clusters ou autres dispositifs, où la vraie intonation fausse est parfaite.
D. J. - Autrement dit, vous avez remplacé des difficultés non rentables par des difficultés rentables?

P. B. - Voilà. Grâce à l'expérience.

D. J. - Est-ce que ce pourrait être une définition de la composition?

P. B. - Non, c'est trop limité. Il faut se méfier aussi de dire « il faut que ce soit facile, il faut que ce soit possible dans une répétition, etc. ». J'exagère, bien sûr, il faut que cela soit possible - mais quand même je trouve qu'il faut toujours placer la barre assez haut. Parce que, lorsque je considère toutes les œuvres que j'ai composées, elles sont encore difficiles, mais la difficulté diminue au fur et à mesure. Par exemple je vois même certains passages d'Éclats multiples : quand on a de bons artistes, qui sont non seulement de bons instrumentistes, mais aussi de bons musiciens, on y arrive très bien. Ce qui était très difficile il y a une trentaine d'années, quand j'ai écrit l'œuvre. Et quand nous répétons Le Marteau avec les musiciens de l'Ensemble intercontemporain, on a besoin d'une seule répétition, ou une et demie au maximum. Quand je me rappelle ce que c'était en 1955, et le mal que s'est donné Rosbaud pour travailler chaque partie...

D. J. - Il semble bien évidemment que dans le champ musical, les questions proprement scripturaires intégrant ou n' intégrant pas la notion d' accident soient finalement un peu secondes, moins importantes que tous les problèmes liés à l'exécution?

P. B. - Oui.

D. J. - Parce que la musique, c'est l'exécution.

P. B. - La musique n'existe que parce que vous l'exécutez; elle existe bien pour les gens qui peuvent lire une partition, mais combien de gens peuvent lire une partition par rapport aux gens qui peuvent l'entendre?

D. J. - Par exemple j' imagine qu' une auvre comme la Sonatine pour flûte et piano de 1946 devait apparaître à ses 
interprètes d'alors - fussent-ils éminents, comme Severino Gazelloni et David Tudor - comme une succession d'accidents de jeu possible, alors qu' aujourd' hui Sophie Cherrier et Pierre-Laurent Aimard jouent cela comme du Mozart!

P. B. - Oui, c'est vrai. Je pense qu'il y a une façon nouvelle de concevoir l'ensemble, la rythmique aussi, la pulsion rythmique... Cela ne concerne pas seulement la musique contemporaine. En 1945-1946, on pouvait constater que Le Sacre du printemps était encore une œuvre très difficile pour les orchestres. Ils restaient très prudents, et même plus que prudents. Maintenant, c'est un cheval de bataille pour tous les grands orchestres!

\section{Euvres de Pierre Boulez citées dans l'entretien}

\section{Le Soleil des eaux}

Texte de René Char. Versions successives : première version de concert pour soprano, ténor, basse et orchestre de chambre (1950) ; version définitive : soprano, chœur mixte et orchestre (1965).

Le Visage nuptial

Texte de René Char. Versions successives : première version pour soprano, alto et quatre instruments (1946-1947) ; version définitive pour soprano, mezzo, chœurs et orchestre (1985-1989).

III Sonate pour piano

Version première complète (1957). Seuls deux mouvements sont considérés comme terminés. «Constellation-Miroir » est un des deux parcours possibles du troisième mouvement, l'autre étant «Constellation».
Pli selon pli

Portrait de Mallarmé. Don [soprano et orchestre (1960-1962)] ; Trois Improvisations sur Mallarmé [soprano et diverses formations instrumentales (1957-1959)] ; Tombeau [soprano et orchestre (1959-1962)]. Version définitive : 1984.

Cummings ist der Dichter

Textes de E. E. Cummings. Double-chœur (seize voix) et ensemble instrumental (vingt-quatre instrumentistes). 1970. Version révisée (1987).

\section{Le Marteau sans maître}

Textes de René Char. Alto et six instrumentistes. 1953, révision 1957.

Poésie pour pouvoir

Texte d'Henri Michaux. Bande magnétique et trois groupes orchestraux (1958). Partition retirée après la création.

Éclat, pour quinze instruments (1965); Éclat-Multiples, pour vingt-sept instruments (1966). Considéré comme inachevé.

Explosante-fixe

Flûte-MIDI, ensemble de chambre et Live-Elektronik. Dernier état de différentes versions sous le même titre, de 1971 à 1993.

Structures pour deux pianos

Premier Livre (1952). Second Livre (1962).

Incises/Sur incises

Incises [pièce pour piano (1993-1999)] ; Sur incises [trois pianos, trois harpes, trois percussions (1996-1998)].

Répons

Six solistes, ensemble instrumental, Live-Elektronik (1981-1996).

Dérive I

Pour flûte, clar A, vibraphone, piano, violon et violoncelle (1984).

Dérive II

Onze instrumentistes. Diverses versions (1988-2006).

Sonatine

Pour flûte et piano (1946).

DominiQue JAMEuX est l'auteur de Pierre Boulez (Paris, Fayard, 1984) et de L'École de Vienne (Paris, Fayard, 2002), ainsi que de monographies sur R. Strauss et A. Berg (Paris, Éditions du Seuil, 1970 et 1980) et de la présentation et la publication des Écrits d'Alban Berg (Paris, Christian Bourgois, 1985). Nombreux textes et conférences en France et à l'étranger. Fellow au « Wissenschaftskolleg zu Berlin » (1996-1997). Producteur à France Culture et à France Musique (1972-2008). Dernier ouvrage paru : Radio (Paris, Fayard, 2009). 